K. BHOI, P. K. RAY

\title{
REPDIGITS AS DIFFERENCE OF TWO FIBONACCI OR LUCAS NUMBERS
}

K. Bhoi, P. K. Ray. Repdigits as difference of two Fibonacci or Lucas numbers, Mat. Stud. 56 (2021), 124-132.

In the present study we investigate all repdigits which are expressed as a difference of two Fibonacci or Lucas numbers. We show that if $F_{n}-F_{m}$ is a repdigit, where $F_{n}$ denotes the $n$-th Fibonacci number, then $(n, m) \in\{(7,3),(9,1),(9,2),(11,1),(11,2),(11,9),(12,11),(15,10)\}$. Further, if $L_{n}$ denotes the $n$-th Lucas number, then $L_{n}-L_{m}$ is a repdigit for $(n, m) \in$ $\{(6,4),(7,4),(7,6),(8,2)\}$, where $n>m$. Namely, the only repdigits that can be expressed as difference of two Fibonacci numbers are 11,33,55,88 and 555; their representations are $11=F_{7}-F_{3}, 33=F_{9}-F_{1}=F_{9}-F_{2}, 55=F_{11}-F_{9}=F_{12}-F_{11}, 88=F_{11}-F_{1}=$ $F_{11}-F_{2}, 555=F_{15}-F_{10}$ (Theorem 2). Similar result for difference of two Lucas numbers: The only repdigits that can be expressed as difference of two Lucas numbers are 11, 22 and 44; their representations are $11=L_{6}-L_{4}=L_{7}-L_{6}, 22=L_{7}-L_{4}, 4=L_{8}-L_{2}$ (Theorem 3).

1. Introduction. The Fibonacci sequence $\left\{F_{n}\right\}_{n \geq 0}$ and the Lucas sequence $\left\{L_{n}\right\}_{n \geq 0}$ are recursively defined by the binary recurrences $F_{n+2}=F_{n+1}+F_{n}, F_{0}=0, F_{1}=1$ and $L_{n+2}=$ $L_{n+1}+L_{n}, L_{0}=2, L_{1}=1$, respectively. The Binet's formulas for both the sequences are given by $F_{n}=\frac{\alpha^{n}-\beta^{n}}{\alpha-\beta}$ and $L_{n}=\alpha^{n}+\beta^{n}$, where $\alpha=\frac{1+\sqrt{5}}{2}$ and $\beta=\frac{1-\sqrt{5}}{2}$ are the roots of the characteristic equation $x^{2}=x+1$. One can observe that for $n \geq 1$,

$$
\alpha^{n-2} \leq F_{n} \leq \alpha^{n-1}, \quad \alpha^{n-1} \leq L_{n} \leq 2 \alpha^{n} .
$$

A natural number $N$ is known as a repdigit if all of its digits are equal. Mathematically, it is in the form $d\left(10^{k}-1\right) / 9$, where $d \in\{1,2, \ldots, 9\}$. For $k=1$, it is a trivial repdigit. Recently, searching of repdigits in linear recurrence sequences have been seen in several papers. For example, Luca [9] proved that 55 and 11 are the largest repdigits in the Fibonacci and Lucas sequences respectively. Marques and Togbé [10] showed that if $F_{n} \cdots F_{n+(k-1)}$ is a repdigit, with at least two digits, then $(k, n)=(1,10)$. In [1], Adegbindin et. al determined all Lucas numbers that are sums of two repdigits. Alahmadi et. al [2] found all the Fibonacci numbers which are concatenation of two repdigits. In [7], Erduvan et. al found all Fibonacci and Lucas numbers which can be written as a difference of two repdigits. Here, they studied the Diophantine equations

$$
F_{k}=d_{1}\left(\frac{10^{n}-1}{9}\right)-d_{2}\left(\frac{10^{n}-1}{9}\right), \quad L_{k}=d_{1}\left(\frac{10^{n}-1}{9}\right)-d_{2}\left(\frac{10^{n}-1}{9}\right),
$$

where $(k, m, n)$ are positive integers with $n \geq 2$ and showed that $F_{11}=89=111-22$ and $L_{18}=5778=6666-888$ are the largest Fibonacci and Lucas numbers which can be written as a difference of two repdigits.

2010 Mathematics Subject Classification:11B39, 11J86, $11 \mathrm{D} 61$.

Keywords: Fibonacci sequence; Lucas sequence; linear forms in logarithms; Baker-Davenport reduction method.

doi:10.30970/ms.56.2.124-132

(C) K. Bhoi, P. K. Ray, 2021 
In this study we search repdigits which can be expressed as a difference of two Fibonacci or Lucas numbers. For this purpose, we consider the following two equations

$$
\begin{aligned}
& F_{n}-F_{m}=d\left(\frac{10^{k}-1}{9}\right), \\
& L_{n}-L_{m}=d\left(\frac{10^{k}-1}{9}\right),
\end{aligned}
$$

with $n>m$ and $1 \leq d \leq 9$.

Assume $k \geq 2$ to avoid trivial solutions.

2. Preliminaries. Baker's theory of linear forms in logarithms of algebraic numbers plays an important role while solving various Diophantine equations. We start by recalling some basic notations and results from algebraic number theory.

Let $\eta$ be an algebraic number with minimal primitive polynomial

$$
f(X)=a_{0}\left(X-\eta^{(1)}\right) \ldots\left(X-\eta^{(k)}\right) \in \mathbb{Z}[X],
$$

where $a_{0}>0$, and $\eta^{(i)}$ 's are conjugates of $\eta$. Then

$$
h(\eta)=\frac{1}{k}\left(\log a_{0}+\sum_{j=1}^{k} \max \left\{0, \log \left|\eta^{(j)}\right|\right\}\right)
$$

is called the logarithmic height of $\eta$. If $\eta=a / b$ is a rational number with $\operatorname{gcd}(a, b)=1$ and $b>1$, then $h(\eta)=\log (\max \{|a|, b\})$. Some properties of the logarithmic height, needed in our proofs, are the following:

$$
h(\eta \pm \gamma) \leq h(\eta)+h(\gamma)+\log 2, \quad h\left(\eta \gamma^{ \pm 1}\right) \leq h(\eta)+h(\gamma), \quad h\left(\eta^{k}\right)=|k| h(\eta) .
$$

With the above notations, Matveev (see [11] or [3, Theorem 9.4]) proved the following result.

Theorem 1. Let $\mathbb{L}$ be an algebraic number field of degree $d_{\mathbb{L}}$. Let $\eta_{1}, \eta_{2}, \ldots, \eta_{l} \in \mathbb{L}$ be positive real numbers and $b_{1}, b_{2}, \ldots, b_{l}$ be non zero integers. If $\Gamma=\prod_{i=1}^{l} \eta_{i}^{b_{i}}-1$ is not zero, then

$$
\log |\Gamma|>-1.4 \cdot 30^{l+3} \cdot l^{4.5} \cdot d_{\mathbb{L}}^{2}\left(1+\log d_{\mathbb{L}}\right)(1+\log D) A_{1} A_{2} \ldots A_{l},
$$

where $D \geq \max \left\{\left|b_{1}\right|,\left|b_{2}\right|, \ldots,\left|b_{l}\right|\right\}$ and $A_{1}, A_{2}, \ldots, A_{l}$ are positive integers such that

$$
A_{j} \geq h^{\prime}\left(\eta_{j}\right)=\max \left\{d_{\mathbb{L}} h\left(\eta_{j}\right),\left|\log \eta_{j}\right|, 0.16\right\} \text { for } j=1, \ldots, l \text {. }
$$

The following result of Baker-Davenport due to Dujella and Pethő [6] is another tool in our proofs. It will be used to reduce the upper bounds of the variables on (1) and (2).

Lemma 1 ([6]). Let $M$ be a positive integer and $p / q$ be a convergent of the continued fraction of the irrational number $\tau$ such that $\tau>6 M$. Let $A, B, \mu$ be some real numbers with $A>0$ and $B>1$. Let $\varepsilon:=\|\mu q\|-M\|\tau q\|$, where $\|$.$\| denotes the distance from the$ nearest integer. If $\varepsilon>0$, then there exists no solution to the inequality

$$
0<|u \tau-v+\mu|<A B^{-w}
$$

in positive integers $u, v, w$ with $u \leq M$ and $w \geq \frac{\log (A q / \varepsilon)}{\log B}$.

When $\mu=0$, we get $\varepsilon<0$. In this case, we cannot apply Lemma 1 . We use the following result due to Legendre. 
Lemma 2 (Legendre, $[4,5]$ ). Let $\lambda$ be a real number and $x, y$ integers such that

$$
\left|\lambda-\frac{x}{y}\right|<\frac{1}{2 y^{2}}
$$

Then $x / y=p_{t} / q_{t}$ is a convergent of $\lambda$. Further, let $M$ and $N$ be nonnegative integers such that $q_{N}>M$. Then putting $a(M)=\max \left\{a_{i}: i=0,1,2, \ldots, N\right\}$, the inequality

$$
\left|\lambda-\frac{x}{y}\right| \geq \frac{1}{(a(M)+2) y^{2}}
$$

holds for all pairs $(x, y)$ of positive integers with $0<y<M$.

The following lemma will also be used in order to prove our subsequent results.

Lemma 3 ([8]). Let $r \geq 1$ and $H>0$ be such that $H>\left(4 r^{2}\right)^{r}$ and $H>L /(\log L)^{r}$. Then

$$
L<2^{r} H(\log H)^{r} .
$$

3. Repdigits as difference of two Fibonacci numbers. Our first result is the following.

Theorem 2. The only repdigits that can be expressed as difference of two Fibonacci numbers are $11,33,55,88$ and 555 . Their representations are

$$
\begin{aligned}
& 11=F_{7}-F_{3}, \quad 33=F_{9}-F_{1}=F_{9}-F_{2}, \quad 55=F_{11}-F_{9}=F_{12}-F_{11}, \\
& 88=F_{11}-F_{1}=F_{11}-F_{2}, \quad 555=F_{15}-F_{10} .
\end{aligned}
$$

Proof. Using Mathematica, we obtain all the solutions of (1) for $n \in[1,200]$ as listed above. From now, assume that $n>200$. The inequality $10^{k-1}<F_{n} \leq \alpha^{n-1}$ implies $k \log 10-3<$ $n \log \alpha$. Using Binet's formula for the Fibonacci sequence, (1) can be written as

$$
\frac{\alpha^{n}-\beta^{n}}{\sqrt{5}}-\frac{\alpha^{m}-\beta^{m}}{\sqrt{5}}=d\left(\frac{10^{k}-1}{9}\right),
$$

which further implies $\frac{\alpha^{n}}{\sqrt{5}}-\frac{d 10^{k}}{9}=\frac{\alpha^{m}}{\sqrt{5}}+\frac{\beta^{n}}{\sqrt{5}}-\frac{\beta^{m}}{\sqrt{5}}-\frac{d}{9}$. Taking absolute values on both sides, we obtain

$$
\left|\frac{\alpha^{n}}{\sqrt{5}}-\frac{d 10^{k}}{9}\right| \leq\left|\frac{\alpha^{m}}{\sqrt{5}}\right|+3 \leq \frac{7 \alpha^{m}}{\sqrt{5}} .
$$

Dividing both sides by $\frac{\alpha^{n}}{\sqrt{5}}$ gives

$$
\left|1-\alpha^{-n} 10^{k}\left(\frac{d \sqrt{5}}{9}\right)\right|<\frac{7}{\alpha^{n-m}} .
$$

We set $\Gamma=1-\alpha^{-n} 10^{k}\left(\frac{d \sqrt{5}}{9}\right)$. We need to show $\Gamma \neq 0$. On the contrary, suppose $\Gamma=0$, then $\alpha^{2 n}=\frac{5 d^{2} 10^{2 k}}{81}$. Since $\frac{5 d^{2} 10^{2 k}}{81} \in \mathbb{Q}, \alpha^{2 n} \in \mathbb{Q}$, which is a contradiction to the fact that $\alpha^{n}$ is irrational for any $n>0$. Therefore, $\Gamma \neq 0$. Now, take

$$
\eta_{1}=\alpha, \eta_{2}=10, \eta_{3}=\frac{d \sqrt{5}}{9}, b_{1}=-n, b_{2}=k, b_{3}=1, l=3,
$$

where $\eta_{1}, \eta_{2}, \eta_{3} \in \mathbb{Q}(\alpha)$ and $b_{1}, b_{2}, b_{3} \in \mathbb{Z}$. Observe that $\mathbb{Q}\left(\eta_{1}, \eta_{2}, \eta_{3}\right)=\mathbb{Q}(\alpha)$, so $d_{\mathbb{L}}=2$. Since $k<n$, we take $D=\max \{n, k, 1\}=n$. The logarithmic heights of $\eta_{1}, \eta_{2}$ and $\eta_{3}$ are calculated as

$$
h\left(\eta_{1}\right)=\frac{\log \alpha}{2}, h\left(\eta_{2}\right)=\log 10 \text { and } h\left(\eta_{3}\right) \leq h(d \sqrt{5})+h(9)<5.2 .
$$


Thus,

$$
\begin{aligned}
& \max \left\{2 h\left(\eta_{1}\right),\left|\log \eta_{1}\right|, 0.16\right\}=\log \alpha=A_{1}, \\
& \max \left\{2 h\left(\eta_{2}\right),\left|\log \eta_{2}\right|, 0.16\right\}=2 \log 10=A_{2}, \\
& \max \left\{2 h\left(\eta_{3}\right),\left|\log \eta_{3}\right|, 0.16\right\}<10.5=A_{3} .
\end{aligned}
$$

Now, applying Theorem 1, yields

$$
\log |\Gamma|>-1.4 \cdot 30^{6} \cdot 3^{4.5} \cdot 2^{2}(1+\log 2)(1+\log n)(\log \alpha)(2 \log 10)(10.5) .
$$

Comparing the above inequality with (4) gives

$$
(n-m) \log \alpha<\log 7+2.3 \cdot 10^{13}(1+\log n)<2.4 \cdot 10^{13}(1+\log n) .
$$

We rewrite (3) to obtain

$$
\frac{\alpha^{n}}{\sqrt{5}}-\frac{\alpha^{m}}{\sqrt{5}}-\frac{d 10^{k}}{9}=\frac{\beta^{n}}{\sqrt{5}}-\frac{\beta^{m}}{\sqrt{5}}-\frac{d}{9} .
$$

Taking absolute values on both sides, we have

$$
\left|\frac{\alpha^{n}}{\sqrt{5}}-\frac{\alpha^{m}}{\sqrt{5}}-\frac{d 10^{k}}{9}\right| \leq 3 .
$$

Dividing both sides by $\frac{\alpha^{n}}{\sqrt{5}}\left(1-\alpha^{m-n}\right)$ implies

$$
\left|1-\alpha^{-n} 10^{k}\left(\frac{d \sqrt{5}}{9\left(1-\alpha^{m-n}\right)}\right)\right|<\frac{7}{\alpha^{n}} .
$$

Now, set

$$
\Gamma^{\prime}=1-\alpha^{-n} 10^{k}\left(\frac{d \sqrt{5}}{9\left(1-\alpha^{m-n}\right)}\right) .
$$

In a similar manner, one can check that $\Gamma^{\prime} \neq 0$. As before, we have $h\left(\eta_{1}\right)=h(\alpha)=\frac{\log \alpha}{2}$ and $h\left(\eta_{2}\right)=h(10)=\log 10$. Let $\eta_{3}=\left(\frac{d \sqrt{5}}{9\left(1-\alpha^{m-n}\right)}\right)$. Then,

$$
\begin{gathered}
h\left(\eta_{3}\right) \leq h(d \sqrt{5})+h\left(9\left(1-\alpha^{m-n}\right)\right) \leq \\
\leq 2 \log 9+\log (\sqrt{5})+(n-m) \frac{\log \alpha}{2}+\log 2<5.9+(n-m) \frac{\log \alpha}{2} .
\end{gathered}
$$

Hence, from (5) we obtain

$$
h\left(\eta_{3}\right)<1.3 \cdot 10^{13}(1+\log n) .
$$

Thus, we take $A_{3}=2.6 \cdot 10^{13}(1+\log n)$. By virtue of Theorem 1 $\log |\Gamma|>-1.4 \cdot 30^{6} \cdot 3^{4.5} \cdot 2^{2}(1+\log 2)(1+\log n)(\log \alpha)(2 \log 10)\left(2.6 \cdot 10^{13}(1+\log n)\right)$. 
Comparing the above inequality with (6) gives

$$
n \log \alpha<\log 7+5.6 \cdot 10^{25}(1+\log n)^{2}<5.7 \cdot 10^{25}(1+\log n)^{2} .
$$

With the notations of Lemma 3, we take $r=2, L=n$ and $H=\frac{5.7 \cdot 10^{25}}{\log \alpha}$ to get

$$
n<2^{2}\left(\frac{5.7 \cdot 10^{25}}{\log \alpha}\right)\left(\log \left(\frac{5.7 \cdot 10^{25}}{\log \alpha}\right)\right)^{2}<1.8 \cdot 10^{30}
$$

Now, we reduce the bound by using the Baker-Davenport reduction method due to Dujella and Pethô. Let

$$
\Lambda=-n \log \alpha+k \log 10+\log \left(\frac{d \sqrt{5}}{9}\right)
$$

The inequality (4) can be written as

$$
\left|e^{\Lambda}-1\right|<\frac{7}{\alpha^{n-m}} .
$$

Observe that $\Lambda \neq 0$ as $e^{\Lambda}-1=\Gamma \neq 0$. Assuming $n-m \geq 6$, the right-hand side in the above inequality is at most $\frac{448}{(1+\sqrt{5})^{6}}<\frac{1}{2}$. The inequality $\left|e^{z}-1\right|<y$ for real values of $z$ and $y$ implies $z<2 y$. Thus, we get $|\Lambda|<\frac{14}{\alpha^{n-m}}$, which implies that

$$
\left|-n \log \alpha+k \log 10+\log \left(\frac{d \sqrt{5}}{9}\right)\right|<\frac{14}{\alpha^{n-m}} .
$$

Dividing both sides by $\log \alpha$ gives

$$
\left|k\left(\frac{\log 10}{\log \alpha}\right)-n+\left(\frac{\log (d \sqrt{5} / 9)}{\log \alpha}\right)\right|<\frac{30}{\alpha^{n-m}} .
$$

To apply Lemma 1, let

$$
u=k, \tau=\left(\frac{\log 10}{\log \alpha}\right), v=n, \mu=\left(\frac{\log (d \sqrt{5} / 9)}{\log \alpha}\right), A=30, B=\alpha, w=n-m .
$$

We can take $M=1.8 \cdot 10^{30}$. We find $q_{61}=25723116487424714265759180025093$, the denominator of 61-th convergent of $\tau$ exceeds $6 M$ with $0<\varepsilon:=\left\|\mu q_{61}\right\|-M\left\|\tau q_{61}\right\|=$ 0.221688. Applying Lemma 1 to the inequality (7) for $1 \leq d \leq 9$, we get $n-m \leq 160$. Now, for $n-m \leq 160$, put

$$
\Lambda^{\prime}=-n \log \alpha+k \log 10+\log \left(\frac{d \sqrt{5}}{9\left(1-\alpha^{m-n}\right)}\right) .
$$

The inequality (6) can be written as $\left|e^{\Lambda^{\prime}}-1\right|<\frac{7}{\alpha^{n}}$. Observe that $\Lambda^{\prime} \neq 0$ as $e^{\Lambda^{\prime}}-1=\Gamma \neq 0$. Assuming $n \geq 6$, the right-hand side in the above inequality is at most $\frac{448}{(1+\sqrt{5})^{6}}<\frac{1}{2}$. The inequality $\left|e^{z}-1\right|<y$ for real values of $z$ and $y$ implies $z<2 y$. Thus, we get $\left|\Lambda^{\prime}\right|<\frac{14}{\alpha^{n}}$, which implies that

$$
\left|-n \log \alpha+k \log 10+\log \left(\frac{d \sqrt{5}}{9\left(1-\alpha^{m-n}\right)}\right)\right|<\frac{14}{\alpha^{n}} .
$$


Dividing both sides by $\log \alpha$ gives

$$
\left|k\left(\frac{\log 10}{\log \alpha}\right)-n+\left(\frac{\log \left(d \sqrt{5} /\left(9\left(1-\alpha^{m-n}\right)\right)\right)}{\log \alpha}\right)\right|<\frac{30}{\alpha^{n}} .
$$

Let

$$
u=k, \tau=\left(\frac{\log 10}{\log \alpha}\right), v=n, \mu=\frac{\log \left(d \sqrt{5} /\left(9\left(1-\alpha^{m-n}\right)\right)\right)}{\log \alpha}, A=30, B=\alpha, w=n .
$$

Choose $M=1.8 \cdot 10^{30}$. We find $q_{70}=20589775267077186120582738407535948$, the denominator of 70-th convergent of $\tau$ exceeds $6 M$ with $0<\varepsilon:=\left\|\mu q_{70}\right\|-M\left\|\tau q_{70}\right\|=$ 0.000138226 . Applying Lemma 1 to the inequality (8) for $1 \leq d \leq 9$ and $n-m \leq 160$, we get $n \leq 189$. This contradicts the assumption that $n>200$.

4. Repdigits as difference of two Lucas numbers. Our second result is the following.

Theorem 3. The only repdigits that can be expressed as difference of two Lucas numbers are 11, 22 and 44. Their representations are

$$
11=L_{6}-L_{4}=L_{7}-L_{6}, \quad 22=L_{7}-L_{4}, \quad 4=L_{8}-L_{2} .
$$

Proof. Using Mathematica, we obtain all the solutions of (2) for $n \in[1,200]$ as listed above. From now, assume that $n>200$. The inequality $10^{k-1}<L_{n} \leq 2 \alpha^{n}$ implies $k \log 10-2<$ $n \log \alpha$. Using Binet's formula for the Lucas sequence, (2) can be written as

$$
\alpha^{n}+\beta^{n}-\alpha^{m}-\beta^{m}=d\left(\frac{10^{k}-1}{9}\right) .
$$

We write (9) to obtain

$$
\alpha^{n}-\frac{d 10^{k}}{9}=\alpha^{m}+\beta^{m}-\beta^{n}-\frac{d}{9} .
$$

Taking absolute values and dividing by $\alpha^{n}$ on both sides, we get

$$
\left|1-\alpha^{-n} 10^{k}\left(\frac{d}{9}\right)\right|<\frac{4}{\alpha^{n-m}} .
$$

We set $\Gamma=1-\alpha^{-n} 10^{k}\left(\frac{d}{9}\right)$. If $\Gamma=0$, then $\alpha^{2 n}=\frac{d^{2} 10^{2 k}}{81}$. It is easily checked that $\alpha^{n}$ is irrational for every positive integer $n$. The irrationality of $\alpha^{n}$ immediately implies the non-vanishing of $\Gamma$.

Take $\eta_{1}=\alpha, \eta_{2}=10, \eta_{3}=\frac{d}{9}, b_{1}=-n, b_{2}=k, b_{3}=1, l=3$, where $\eta_{1}, \eta_{2}, \eta_{3} \in \mathbb{Q}(\alpha)$ and $b_{1}, b_{2}, b_{3} \in \mathbb{Z}$. Observe that $\mathbb{Q}\left(\eta_{1}, \eta_{2}, \eta_{3}\right)=\mathbb{Q}(\alpha)$, so $d_{\mathbb{L}}=2$. Since $k<n$, we take $D=\max \{n, k, 1\}=n$. The logarithmic heights of $\eta_{1}, \eta_{2}$ and $\eta_{3}$ are calculated as

$$
h\left(\eta_{1}\right)=\frac{\log \alpha}{2}, h\left(\eta_{2}\right)=\log 10 \text { and } h\left(\eta_{3}\right) \leq h(d)+h(9)<4.4 .
$$

Thus, we take $A_{1}=\log \alpha, A_{2}=2 \log 10$ and $A_{3}=8.8$. Applying Theorem 1, we have

$$
\log |\Gamma|>-1.4 \cdot 30^{6} \cdot 3^{4.5} \cdot 2^{2}(1+\log 2)(1+\log n)(\log \alpha)(2 \log 10)(8.8) .
$$


Comparing the above inequality with (4) gives

$$
(n-m) \log \alpha<\log 4+1.9 \cdot 10^{13}(1+\log n)<2 \cdot 10^{13}(1+\log n) .
$$

We rewrite (9) to obtain

$$
\alpha^{n}-\alpha^{m}-\frac{d 10^{k}}{9}=\beta^{m}-\beta^{n}-\frac{d}{9} .
$$

Taking absolute values and dividing by $\alpha^{n}\left(1-\alpha^{m-n}\right)$ on both sides, we get

$$
\left|1-\alpha^{-n} 10^{k}\left(\frac{d}{9\left(1-\alpha^{m-n}\right)}\right)\right|<\frac{3}{\alpha^{n}} .
$$

Put

$$
\Gamma^{\prime}=1-\alpha^{-n} 10^{k}\left(\frac{d}{9\left(1-\alpha^{m-n}\right)}\right) .
$$

It is easily checked that $\Gamma^{\prime} \neq 0$. As before, we have $h\left(\eta_{1}\right)=\frac{\log \alpha}{2}$ and $h\left(\eta_{2}\right)=\log 10$. Let $\eta_{3}=\left(\frac{d}{9\left(1-\alpha^{m-n}\right)}\right)$. Then,

$$
h\left(\eta_{3}\right) \leq h(d)+h\left(9\left(1-\alpha^{m-n}\right)\right) \leq 2 \log 9+(n-m) \frac{\log \alpha}{2}+\log 2<5.1+(n-m) \frac{\log \alpha}{2} .
$$

Hence, from $(12)$ we obtain $h\left(\eta_{3}\right)<1.1 \cdot 10^{13}(1+\log n)$. Thus, we take $A_{3}=2.2 \cdot 10^{13}(1+$ $\log n)$. By virtue of Theorem 1

$$
\log |\Gamma|>-1.4 \cdot 30^{6} \cdot 3^{4.5} \cdot 2^{2}(1+\log 2)(1+\log n)(\log \alpha)(2 \log 10)\left(2.2 \cdot 10^{13}(1+\log n)\right) .
$$

Comparing the above inequality with (13) gives

$$
n \log \alpha<\log 3+4.8 \cdot 10^{25}(1+\log n)^{2}<4.9 \cdot 10^{25}(1+\log n)^{2} .
$$

With the notations of Lemma 3, we take $r=2, L=n$ and $H=\frac{4 \cdot 9 \cdot 10^{25}}{\log \alpha}$. Applying the lemma, we have

$$
n<2^{2}\left(\frac{4.9 \cdot 10^{25}}{\log \alpha}\right)\left(\log \left(\frac{4.9 \cdot 10^{25}}{\log \alpha}\right)\right)^{2}<1.5 \cdot 10^{30}
$$

Now, we reduce the bound by using the Baker-Davenport reduction method due to Dujella and Pethó. Let $\Lambda=-n \log \alpha+k \log 10+\log \left(\frac{d}{9}\right)$. The inequality (11) can be written as $\left|e^{\Lambda}-1\right|<\frac{4}{\alpha^{n-m}}$. Observe that $\Lambda \neq 0$ as $e^{\Lambda}-1=\Gamma \neq 0$. Assuming $n-m \geq 5$, the right-hand side in the above inequality is at most $\frac{128}{(1+\sqrt{5})^{5}}<\frac{1}{2}$. The inequality $\left|e^{z}-1\right|<y$ for real values of $z$ and $y$ implies $z<2 y$. Thus, we get $|\Lambda|<\frac{8}{\alpha^{n-m}}$, which implies that

$$
\left|-n \log \alpha+k \log 10+\log \left(\frac{d}{9}\right)\right|<\frac{8}{\alpha^{n-m}} .
$$

Dividing both sides by $\log \alpha$ gives

$$
\left|k\left(\frac{\log 10}{\log \alpha}\right)-n+\left(\frac{\log (d / 9)}{\log \alpha}\right)\right|<\frac{17}{\alpha^{n-m}} .
$$


Let

$$
u=k, \tau=\left(\frac{\log 10}{\log \alpha}\right), v=n, \mu=\left(\frac{\log (d / 9)}{\log \alpha}\right), A=17, B=\alpha, w=n-m .
$$

We can take $M=1.5 \cdot 10^{30}$. We find $q_{62}=28291878740422696593104794201645$ satisfies $q>6 M$ with $\varepsilon:=0.0247517$. Applying Lemma 1 to the inequality (14) for $1 \leq d \leq 8$, we get $n-m \leq 164$.

For the case $d=9$, we have that $\mu(d)=0$. In this case, we apply Lemma 2 . The inequality (14) can be rewritten as

$$
\left|\frac{\log 10}{\log \alpha}-\frac{n}{k}\right|<\frac{17}{k \alpha^{n-m}}<\frac{1}{2 k^{2}},
$$

because $k<1.5 \cdot 10^{30}=M$. It follows from Lemma 2 that $\frac{n}{k}$ is a convergent of $\frac{\log 10}{\log \alpha}$. So $\frac{n}{k}$ is of the form $p_{t} / q_{t}$ for some $t=0,1,2, \ldots, 62$. Thus,

$$
\frac{1}{(a(M)+2) k^{2}} \leq\left|\frac{\log 10}{\log \alpha}-\frac{n}{k}\right|<\frac{17}{k \alpha^{n-m}} .
$$

Since $a(M)=\max \left\{a_{i}: i=0,1,2, \ldots, 62\right\}=106$, we get

$$
n-m \leq \frac{\log \left(17 \cdot\left(1.5 \cdot 10^{30}\right) \cdot 108\right)}{\log \alpha} \leq 160 .
$$

Thus $n-m \leq 164$ in both cases.

Now, for $n-m \leq 164$, put

$$
\Lambda^{\prime}=-n \log \alpha+k \log 10+\log \left(\frac{d}{9\left(1-\alpha^{m-n}\right)}\right) .
$$

The inequality (13) can be written as $\left|e^{\Lambda^{\prime}}-1\right|<\frac{3}{\alpha^{n}}$. Observe that $\Lambda^{\prime} \neq 0$ as $e^{\Lambda^{\prime}}-1=\Gamma \neq 0$. Assuming $n-m \geq 4$, the right-hand side in the above inequality is at most $\frac{48}{(1+\sqrt{5})^{4}}<\frac{1}{2}$. The inequality $\left|e^{z}-1\right|<y$ for real values of $z$ and $y$ implies $z<2 y$. Thus, we get $\left|\Lambda^{\prime}\right|<\frac{6}{\alpha^{n}}$, which implies that

$$
\left|-n \log \alpha+k \log 10+\log \left(\frac{d}{9\left(1-\alpha^{m-n}\right)}\right)\right|<\frac{6}{\alpha^{n}} .
$$

Dividing both sides by $\log \alpha$ gives

$$
\left|k\left(\frac{\log 10}{\log \alpha}\right)-n+\left(\frac{\log \left(d /\left(9\left(1-\alpha^{m-n}\right)\right)\right)}{\log \alpha}\right)\right|<\frac{13}{\alpha^{n}} .
$$

To apply Lemma 1, let

$$
u=k, \tau=\left(\frac{\log 10}{\log \alpha}\right), v=n, \mu=\frac{\log \left(d /\left(9\left(1-\alpha^{m-n}\right)\right)\right)}{\log \alpha}, A=13, B=\alpha, w=n .
$$

Choose $M=1.5 \cdot 10^{30}$. We find $q_{70}=20589775267077186120582738407535948$ satisfies $q>6 M$ with $\varepsilon:=0.000607235$. Applying Lemma 1 to the inequality (15) for $1 \leq d \leq 9$ and $n-m \leq 164$, we get $n \leq 184$. This contradicts the assumption that $n>200$. 


\section{REFERENCES}

1. C. Adegbindin, F. Luca, A. Togbé, Lucas numbers as sums of two repdigits, Lith. Math. J., 59 (2019), 295-304.

2. A. Alahmadi, A. Altassan, F. Luca, H. Shoaib, Fibonacci numbers which are concatenation of two repdigits, Quaest. Math., (2019), https://doi.org/10.2989/16073606.2019.1686439.

3. Y. Bugeaud, M. Mignotte, S. Siksek, Classical and modular approaches to exponential Diophantine equations I. Fibonacci and Lucas perfect powers, Ann. of Math. (2), 163 (2006), 969-1018.

4. H. Cohen, A course in computational algebraic number thoery, Springer, New York, 1993.

5. H. Cohen, Number theory. Volume I: Tools and Diophantine equations, Springer, New York, 2007.

6. A. Dujella, A. Pethő, A generalization of a theorem of Baker and Davenport, Quart. J. Math. Oxford Ser., 49 (1998), 291-306.

7. F. Erduvan, R. Keskin, F. Luca, Fibonacci and Lucas numbers as difference of two repdigits, Rend. Circ. Mat. Palermo, II. Ser, (2021) https://doi.org/10.1007/s12215-021-00645-3.

8. S. Gúzman Sánchez, F. Luca, Linear combinations of factorials and s-units in a binary recurrence sequence, Ann. Math. du Qué, 38 (2014), 169-188.

9. F. Luca, Fibonacci and Lucas numbers with only one distinct digit, Port. Math., 57 (2000), 243-254.

10. D. Marques, A. Togbé, On repdigits as product of consecutive Fibonacci numbers, Rend. Istit. Mat. Univ. Trieste, 44 (2012), 393-397.

11. E.M. Matveev, An explicit lower bound for a homogeneous rational linear form in the logarithms of algebraic numbers II, Izv. Ross. Akad. Nauk Ser. Mat., 64 (2000), 125-180. Translation in Izv. Math. 64 (2000), 1217-1269.

Department of Mathematics

Sambalpur University, Jyoti Vihar, Burla, India kisanbhoi.95@suniv.ac.in

Department of Mathematics

Sambalpur University, Jyoti Vihar, Burla, India

prasantamath@suniv.ac.in

Received 07.09.2021

Revised 13.11.2021 